\title{
A Novel Control Strategy for Multiple Switches Based on Electro-Rheological Fluid
}

\author{
Luning XU ${ }^{1,2, a}$, Li HAN ${ }^{1,2, b}$ and Junbiao LIU $^{1,2, c}$ \\ ${ }^{1}$ Nano-micro Technology Department, Institute of Electrical Engineering, Chinese Academy of \\ Sciences, Beijing,100190, China \\ ${ }^{2}$ Beijing Key Laboratory of Bioelectromagnetism, Beijing,100190, China \\ axuluning@mail.iee.ac.cn, ${ }^{\mathrm{b}}$ lihan@mail.iee.ac.cn, ${ }^{\mathrm{C}}$ liujb@mail.iee.ac.cn
}

\begin{abstract}
Keywords: Control Strategy, Switches Matrix, Electro-rheological fluid, Mechatronic Embedded System.
\end{abstract}

\begin{abstract}
A compact or portable mechatronic embedded system application has a strictly demands of volume and/or power consuming. Comparing with electronic parts, switches and/or actuators take the most proportion of volume or weight and power consuming of the whole system. A novel method of connecting multiple switches/actuators built by using electro-rheological fluid and a pair of governing electrodes and their control strategy are introduced in this paper. By using this method, the numbers of control components are changed from $\mathrm{N}$ times $\mathrm{M}$ to $\mathrm{N}$ plus $\mathrm{M}$, the volume and weight reduced largely.A series structure is used in the application on multi-line Braille electro-book for blind people as a demonstration of this novel method. It provides a novel solution of shrinking system for a compact or portable mechatronic embedded system application.
\end{abstract}

\section{Introduction}

Generally, a compact or portable mechatronic embedded system application has a strictly demands of volume and/or power consuming [1-3]. And also, these kinds of systems have lots of switches or actuators in some applications $[4,5]$. A switch or actuator has two states, "on" and 'off'. In order to implement these two states, a control component is necessary, which provides two kinds of signals and powers, to drive switch and to fulfill these two states of the switch. So the numbers of control components are the same as that of switches or actuators.

A power module such as MOSFETs or IGBTs is often as the control component. The usage of MOSFETs or IGBTs depends on the driven power of the switch or actuator they serve. When the numbers of switches are huge in the application, the numbers of their control components are at the same level. A large room is needed to hold these switches and their control components, and the driven modules will consume a lot power. The volume and the consuming power of these switches and their control components will bring problems of space shortage and heat sink to the system designer.

A novel method of connecting multiple switches or and their control strategy is introduced in this paper. This method is propitious to an application which the volume of switch or actuator is comparative smaller than that of their control components. By using this method, the numbers of control components are decreased, so the volume and consuming power of the whole system are reduced largely. If the original numbers of control components can be factorized by $\mathrm{N}$ times $\mathrm{M}, \mathrm{N}$ and $\mathrm{M}$ are both of integer, by using this method, the numbers of control components are down to $\mathrm{N}$ plus M. A series structure is applied in switch connecting, so the numbers of switches are double.

An application on multi-line Braille electro-book for blind people is shown as a demonstration of this novel method of connecting switches and their control strategy. The switch used in the demonstration is built by using electro-rheological fluid and a pair of governing electrodes, which takes a very little space.

This method provides a novel solution for compact embedded system by using small, high density switches, which needs to reduce the numbers and volume of control components to meet the demands of room and power consuming. 


\section{Nomenclature}

$\mathrm{N}$ numbers of row given by factorization of the total numbers of control components; $\mathrm{M}$ numbers of column given by factorization of the total numbers of control components;

\section{Structure of Switches}

The schematic diagram of series structure of switches is shown in Fig. 1.

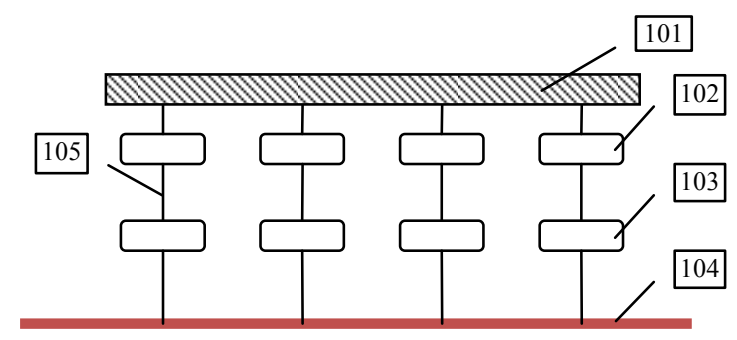

Fig. 1. Schematic diagram of seriers structure of switches

The numbers in Fig. 1 denote input port (101), the first switch of the series structure (102), the second switch of the series structure (103), output port (104), and wire or channel linking these two switches (105) separately.

These two switches serve as one switch. This series structure has the same principle of two input AND gate. Only when these two switches are 'ON' at the same time, the output of this series structure is ' $\mathrm{ON}$ '. Otherwise, the output of this series structure is 'OFF'.

The switch mentioned in the paper means one or a set of unit which can fulfill the function of showing two different states, such as 'ON' and 'OFF' for a power module or 'GO THROUGH' and 'SHUT DOWN' for a valve. In a specific application, the switch may be a power module or a valve. The most important characteristic of the switch for using this connecting method is of small volume, lower power consuming and low cost. Or the reducing of volume, power consuming and cost by using this method cannot offset the increasing part by doubling the switches.

The planform schematic diagram of switches array connecting is shown in Fig. 2. The numbers in Fig. 2 denote series structure of switches (201), the row control line of the series structure (202), and the column control line of the series structure (203) separately.

As it was known, each set of series structure of switches consists of two independent switches. The row control line connects one of these two switches, it was named by 'row switch'; another switch connects the column control line individually, which is named by 'column switch'.

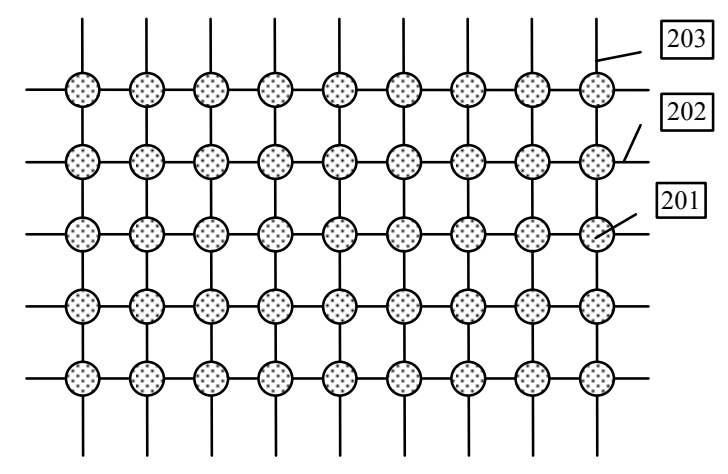

Fig. 2. Planform Schematic Diagram of Switches Array Connecting After Factorization of Original Switches Numbers

The original numbers of control components, before the switches were doubled, can be factorized by $\mathrm{N}$ times $\mathrm{M}, \mathrm{N}$ is a up round integer, which denotes the numbers of row, and $\mathrm{M}$ is also a up round integer, which denotes the numbers of column. Then the row control line is used to link 
each of the 'row switch' of entire columns, and the column control line is used to link each of the 'column switch' of entire rows. Each control line links to one control component. The total numbers of control components are equal to N plus $\mathrm{M}$.

By using this connecting method, a determinant of series switches structure was established, as shown in Fig. 2. The coordinate of each of series switches structure in the determinant is unique. The control strategy is shown as follows.

\section{Control Strategy of Switches}

As mentioned above, the series structure of switches has a function of AND gate, so it becomes possible to fulfill one to one control, which means each of the series switches structure in this determinant can be managed independently by using this connecting method.

When a row control line is assigned a state of ' $\mathrm{ON}$ ', every 'row switch' on this control line will be the state of ' $\mathrm{ON}$ ', and the final state of each series structure is determined by the state of its column control line, namely the state of its 'column switch'. If the state of its 'column switch' is 'ON', the final state of this series structure is ' $O N$ ', the input of the series structure of switches can go through the series structure. Or it will be 'OFF', the series structure has no output. When a row control line is assigned a state of 'OFF', each series structure linking with this line has no output; the states of previous output of these series structures are not changed.

If only one row control line was assigned a state of ' $\mathrm{ON}$ ' among $\mathrm{N}$ row control lines, the others keep on the state of 'OFF' at the moment, and each of their column control lines of this row control line was assigned the state of what it should be, a result will be given as it will. A scanning of $\mathrm{N}$ row control line can be run in turn. When the row control line is ' $O N$ ', states of their column control lines will be effective; the outputs of these series structures can be altered. Final results of entire determinant of series structure will be shown after a whole scanning.

The row control line is as a trigger, and their M column control lines of this row control line are as a set of control data, it is one dimension array; the numbers of its element are M. The combination of the scanning of row control line, the control data from their column control line and series structure of switches contributes to the achievement of managing multiple switches with less control components.

\section{A Demonstration Based on Electro-rheological Fluid Switches}

An application on multi-line Braille electro-book for blind people is shown as a demonstration of this novel method of connecting switches and their control strategy in this part.

The electro-rheological (ER) fluid is a kind of fluid, which has fluidity at normal state; it also has an ability of state alternation with a governing electric field. When a governing electric field is applied, the ER fluid changes its state, turning into a 'solid like' state; it appears a resistance to shear from outside. The more the governing electric field increases, the larger the ability of resisting shear from outside gets.

The switch based on ER fluid has a pair of governing electrodes and a channel for the ER fluid, its section plan is shown in Fig. 3.

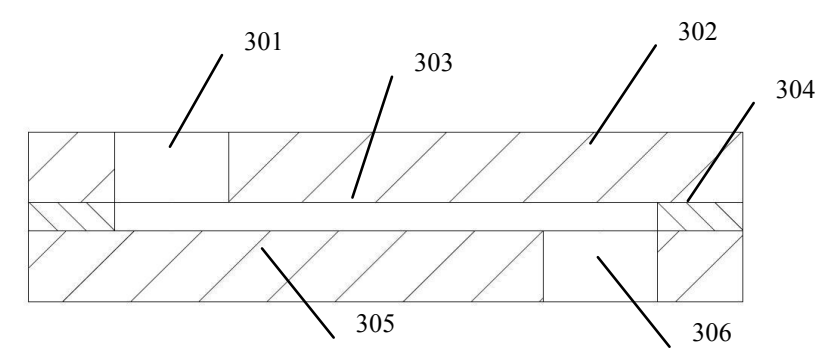

Fig. 3. Section plan of a type of switch based on electro-rheological fluid 
The numbers in Fig. 3 denote output of the switch based on ER fluid (301), anode of the governing electrodes (302), ER fluid channel (303), insulator between electrodes (304), cathode of the governing electrodes (305), and input of the switch based on ER fluid (306).

When a right voltage is applied to the governing electrodes, the ER fluid turns to 'solid like' state; the switch is 'OFF', the output state of the switch doesn't change. When no voltage is applied to the governing electrodes, the switch is 'ON', the output state of the switch changes according to the input state.

The series structure of switches based on ER fluid consists of two switches as what is shown in Fig. 3. For saving materials and system volume, cathode of the single switch is shared when connects these two switches. The section plan of a type of series structure of switches based on ER fluid is shown in Fig. 4.

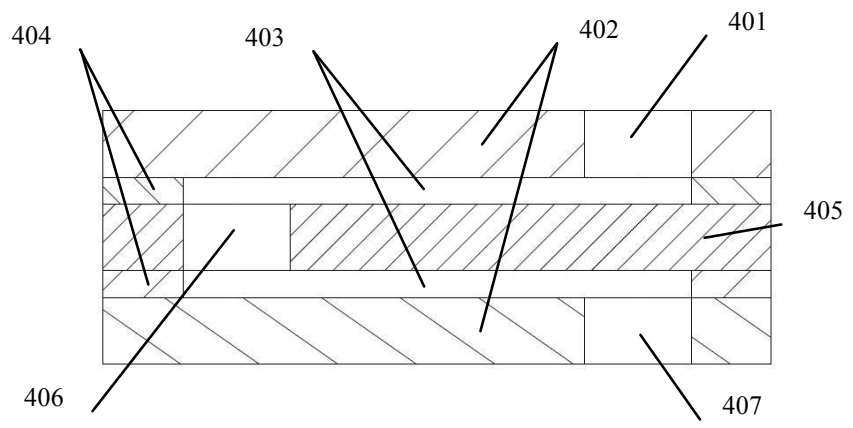

Fig. 4. Section plan of a type of series structure of switches based on electro-rheological fluid

The numbers in Fig. 4 denote output of the series structure of switches based on ER fluid (401), anode of the governing electrodes of each switch (402), ER fluid channel (403), insulator between a pair of electrodes (404), sharing cathode of the governing electrodes of switches (405), linkage channel between these two switches (406) and input of the switch based on ER fluid (407).

As mentioned above, the series structure like a AND gate. Only when these two switches are 'ON', the output of the series structure will change according to its input.

A Braille character is a natural matrix of dots and doesn't need to factorize. One Braille character consists of 6 dots, 2 columns times 3 rows. The distance between dots is not more than $3 \mathrm{~mm}$. For a multiple-line Braille electro-book, there are hundreds of dots, and each dot needs a control component. For example, if there are 30 characters in one line, and there are 25 lines in this electro-book, there are 4,500 dots totally. If one dot matches one control component, 4,500 control components are needed. The system volume and consuming power of control components are considerable. By using this novel method of connecting switches, the numbers of row are 75; the numbers of column are 60. According to either one row or one column just uses one control components, the total numbers of control components are 135. So, the numbers of power switches used to supply the high voltages to the electrodes of ER valves were reduced from 4500 to 135 . The reduction of power consumptions is the sum of those of 4365 power switches. 
The schematic diagram of a demonstration of 4 lines Braille electro-book is shown in Fig. 5.

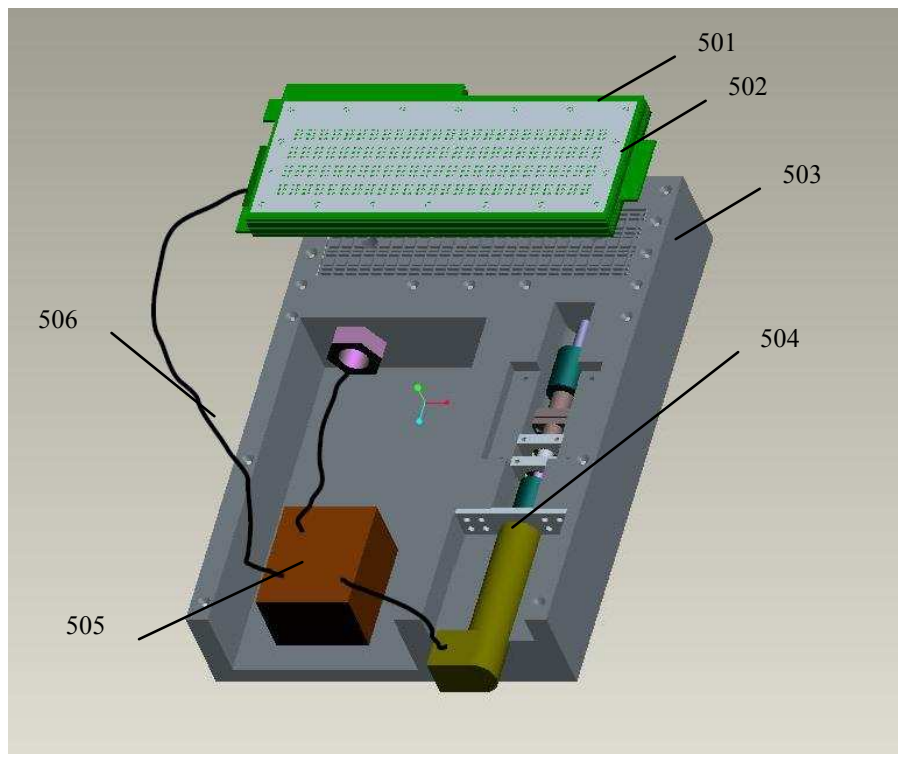

Fig. 5. Schematic diagram of a demonstration of a four-line braille electro-book

The numbers in Fig. 5 denote display layer of Braille character matrix (501), series structure of switches based on ER fluid (502), body of the electro-book (503), driven force module of dots deformation (504), embedded control module of the electro-book (505) and control cables (506).

Because the switch based on ER fluid takes a little room and consuming power, the volume and consuming power of series structure don't increase much. On the contrary, the volume and power consuming reduced by using this novel method of connecting switches are large. For the four-line Braille electro-book, there are 25 characters in one line, and there are 4 lines in this electro-book, there are 600 dots totally. So, the numbers of power switches used to supply the high voltages to the electrodes of ER valves were reduced from 600 to 62 . The reduction of power consumptions is the sum of those of 538 power switches. The final power consumptions are depending on which kinds of power switch do you use and the applications.

\section{Conclusions}

A compact or portable mechatronic embedded system application has a strictly demands of volume and/or power consuming. And also, these kinds of systems have lots of switches or actuators in some applications. A novel solution is provided in this paper. By doubling switches and factorizing the numbers of whole control components, a new control determinant is gotten.

The most important characteristic of the switch for using this connecting method is of small volume, lower power consuming and low cost.

The result by using this novel method is that the numbers of control components the system needs is reduced from the times of row and column of the determinant to the sum of row and column of the determinant.

The combination of the scanning of row control line, the control data from their column control line and series structure of switches contributes to the achievement of managing multiple switches with less control components.

An application on four-line Braille electro-book for blind people is shown as a demonstration of this novel method of connecting switches and their control strategy. There are 25 characters in one line, and there are 4 lines in this electro-book, there are 600 dots totally. The numbers of power switches used to supply the high voltages to the electrodes of ER valves were reduced from 600 to 62 . The reduction of power consumptions is the sum of those of 538 power switches. 


\section{Acknowledgments}

This work was financially supported by Chinese Academy of Sciences (Y060211C31).

\section{References}

[1] Wei Hu, in: Development of Embedded Systems. International Academic Development, Vol. 4 (2010), p. 32-33.

[2] Xizhong Yao, in: Analysis and Reasearch on Embedded Systems and Its Development Trend. Information of Sciences, Vol. 31(2009): p. 88-88.

[3] Xin Liu, Jiaojiao Wang, and Qiang Xu, in: Trend and Current Development of Embedded Systems. China Science and Technology Review, Vol. 21(2010), p. 103-103.

[4] Kocer, A., et al., in: A light-actuated nanovalve derived from a channel protein. Science, Vol. 309(5735) (2005), p. 755-758.

[5] Hisamoto, H., S. Funano, and S. Terabe, Integration of valving and sensing on a capillary-assembled microchip. Analytical Chemistry, Vol. 77(7) (2005), p. 2266-2271. 